\title{
Development and Pathogenicity of the Fungus Crinipellis perniciosa on Interaction with Cacao Leaves
}

\author{
Aruna Kilaru and Karl H. Hasenstein
}

Department of Biology, University of Louisiana, Lafayette 70504.

Accepted for publication 28 September 2004.

\begin{abstract}
Kilaru, A., and Hasenstein, K. H. 2005. Development and pathogenicity of the fungus Crinipellis perniciosa on interaction with cacao leaves. Phytopathology 95:101-107.

We investigated developmental changes in the primary mycelium of Crinipellis perniciosa upon its interaction with immature and mature leaves of Theobroma cacao. On nutritive medium, the primary mycelium grew significantly slower in the presence of host tissue than without host tissue. In the absence of the cacao leaves, incomplete phase transition occurred after 5 days, wherein older hyphae progressed to the dikaryotic state and growing tips remained monokaryotic. Phase transition occurred

leaves, and required 2 weeks on T. cacao callus tissue. The biotrophic mycelia were able to invade immature and mature cacao leaves without open wounds or stomata. Club-shaped hyphal tips and the formation of adhesive structures were induced by cuticle extracts and suggest host recognition. The initial cuticular disintegration at the site of penetration was followed by blister formation and complete digestion of leaves by the primary mycelium. The data suggest specific interactions between host and pathogen that control the onset of the necrotrophic phase of the fungus. The data further indicate that primary mycelium rather than spores can be used to study $C$. perniciosa pathogenicity.
\end{abstract} between 3 and 5 days on mature leaves, 10 and 12 days on meristematic
Additional keywords: host-pathogen interaction.
Crinipellis perniciosa, the causative agent of the witches' broom disease in Theobroma cacao (4), is responsible for major crop losses in South American and Caribbean cocoa plantations. In 1989 the witches' broom disease of cocoa was identified in Bahia, the leading cocoa-growing region in Brazil. In less than 10 years, production shrank from 383,000 tons in $1987-88$ to 150,000 tons estimated for 2002-03. Consequently, Brazil slipped from being the third to the fifth largest cocoa-producing country in the world (14). Various control methods used, including sanitary cultural practices, have not been effective in minimizing damage and incurred high economic costs. The scientific investigations of witches' broom of cacao began in the 1890s and developed considerable knowledge of the biology and epidemiology of the disease (26). However, the mechanisms of host-parasite interaction and the subsequent developmental changes have not been studied.

The pathogen is a hemibiotrophic basidiomycete with two distinguishable phases in its life cycle. The primary, monokaryotic and biotrophic phase is followed by a secondary, dikaryotic but saprophytic stage (25). In suitable environments, the uninucleate basidiospores germinate and develop into relatively wide (5 to $20 \mu \mathrm{m}$ ), convoluted, primary hyphae that invade T. cacao. Incursion by primary hyphae incites major morphological changes in the host tissue, such as stem enlargement, shoot formation, and loss of apical dominance, that gives rise to the characteristic broom appearance and enhanced flowering and fruiting. Subsequent to infection, the tissue becomes necrotic and the primary hyphae undergo phase transition and form thinner $(1.5$ to $3.0 \mu \mathrm{m})$, hyaline, binucleate secondary hyphae with clamp connections that colonize the plant tissues intra- and intercellularly. The saprophytic hyphae eventually produce pink-colored, wide-gilled basidiocarps $\approx 2 \mathrm{~cm}$ in diameter on dead broom tissues that release

Corresponding author: K. H. Hasenstein; E-mail address: hasenstein@louisiana.edu

DOI: 10.1094/PHYTO-95-0101

(C) 2005 The American Phytopathological Society basidiospores, thus completing the life cycle entirely on the host $(8,26,28)$. The factors that regulate the phase transition and cause the death of the host remain unknown. To contain the disease, it is imperative to understand the development and physiology of C. perniciosa.

Past research $(7,24)$ indicated that the monokaryotic primary mycelium is ephemeral and that dikaryotization occurs within 24 to $48 \mathrm{~h}$ on standard agar media. However, Evans (8) reported slow and lasting growth of primary mycelium on cacao callus cultures and concluded that living cacao tissue delays dikaryotization. Because basidiospores were considered to be the only known infective structures $(5,10)$, the only inoculum used in research testing for disease resistance was spores (2). However, generating uncontaminated basidiospores in vitro and the inability to maintain $C$. perniciosa in the primary phase in axenic cultures constrained studies on host-pathogen interactions in witches' broom disease. Furthermore, in previous studies, spores often were inoculated on the abaxial surface, possibly because the entry of germ tubes of $C$. perniciosa through stomata or wounds was observed on pods (30), 4-day-old seedlings (4), and vegetative flushes (9) of T. cacao. To colonize plants, opportunistic fungal parasites are known to depend on natural openings or wounds for invasions. Frias et al. (9) reported a tropic response of germ tubes of C. perniciosa toward stomata on leaves and stems of young flushes and also observed many germ tubes entering a single stoma. Conversely, we observed that, in young leaves, primordial stomata did not attract hyphae. Instead, primary hyphae were capable of direct penetration. Muse et al. (23) showed that the secondary mycelia of $C$. perniciosa entered tissue-cultured cells of cacao independent of stomata and attained initial intercellular growth that developed into intracellular growth at later stages. However, reports on direct penetration of uninjured plant surfaces by $C$. perniciosa $(5,23,29)$ did not describe any specialized structures such as appressoria or haustoria that other pathogens often use to invade the host's tissue (21).

We studied the structural differences of the abaxial and adaxial leaf surfaces of T. cacao and investigated whether the primary 
mycelium of $C$. perniciosa showed preferential growth on either surface. Further, we report that the phase transition in $C$. perniciosa is influenced by the developmental stage of the host tissue and is incomplete in the absence of the host. We observed direct penetration of mature and immature leaf tissue by the primary mycelium with the development of special morphological features and subsequent digestion of the leaves.

\section{MATERIALS AND METHODS}

Inoculum source and application. Mycelial cultures of C. perniciosa were obtained from the American Type Culture Collection (ATCC, strain 64190; permit 53313). Basidiospores were provided by the U.S. Department of Agriculture-Agricultural Research Service (USDA-ARS), Beltsville, MD, and recovered on $0.7 \%$ water agar (WA). We found spores to be highly contaminated, but clean cultures generated from successive subcultures did not indicate any differences in growth rate and colony morphology compared with ATCC cultures on $3.9 \%$ potato dextrose agar (PDA). Regardless of the origin of the cultures, we monitored any possible contamination using PDA as substrate. Mycelia were subcultured every 2 weeks for maintenance. Mycelial plugs were taken from the periphery of 1-week-old PDA cultures.

Plant material and inoculation. T. cacao (common, unidentified clones) plants were maintained in a greenhouse. We selected immature and mature developmental stages of leaves and callus of 4-year-old trees for infection studies. Immature leaves were young, red, tender, and meristematic and the entire leaf was used for inoculation. Mature leaves were green, leathery, and lignified and were cut to fit the petri dish. Leaves were detached, surface sterilized with $0.1 \%$ streptomycin and $1 \%$ dithane, and rinsed in $70 \%$ ethanol prior to inoculation. We generated callus from leaf meristem cultured in modified Murashige and Skoog (MS) medium (22). For all studies, we inoculated adaxial (AD) and abaxial (AB) leaf surfaces with mycelial plugs. For inoculations, the mycelial side of the plugs was placed in contact with the leaf. Pilot studies had shown that the growth rate of the fungus was not affected by which side of the plug was applied (data not shown). Cultures were incubated in a growth chamber (Percival E-54U) at $25^{\circ} \mathrm{C}$ in the dark.

Growth studies. For studies with host tissue, either the AB or $\mathrm{AD}$ sides of eight excised immature or mature cacao leaves were

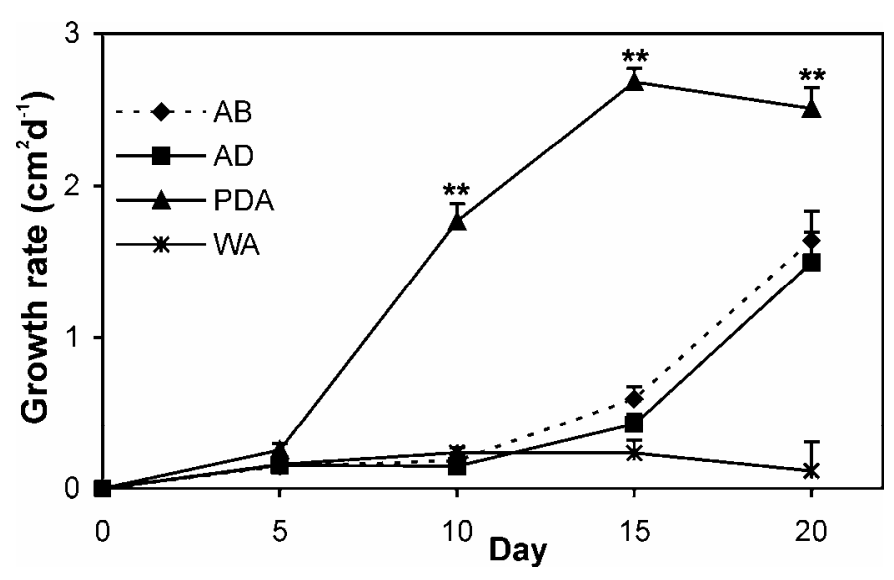

Fig. 1. Growth rate of Crinipellis perniciosa during a 20-day period was significantly affected by the young leaves of Theobroma cacao ( $\mathrm{df}=3, F=$ 139.14, $P<0.0001)$. Interaction with host tissue resulted in a prolonged lag phase regardless of inoculation on the abaxial (AB) or adaxial (AD) leaf surface. Potato dextrose agar (PDA) resulted in the highest growth rate, which declined after 15 days. Water agar (WA) did not support growth. Bars represent standard errors of means and asterisks indicate $P<0.0001(* *)$ between media. placed on PDA medium. A $0.75 \mathrm{~cm}$ mycelial plug was placed next to the central vein. Radial growth was measured 5, 10, 15, and 20 days after inoculation. The surface area of the colony and relative growth rate was calculated from the diameter. Further, we tested whether fungal growth was affected by the position of the inoculum on the leaf. Mycelial plugs were inoculated on the base, middle, and tip regions on the AD surface of young red leaves placed on $0.6 \%$ WA. Radial growth was measured on three replicates after 10 days of inoculation.

Microscopy. Phase transition of $C$. perniciosa was examined after 3, 5, 10, and 15 days. For nuclear staining, mycelium was taken from the peripheral growing, middle, and the innermost region (close to the inoculated plug) and were fixed with $6 \%$ paraformaldehyde in $100 \mathrm{mM}$ PIPES ( $\mathrm{pH}$ 6.8) for $45 \mathrm{~min}$ and rinsed with distilled water. The specimens were stained in $0.01 \%$ calcofluor and $0.1 \%$ 4',6-diamidino-2-phenylindole (DAPI) in water. After $5 \mathrm{~min}$ of incubation in the dark, the samples were washed (three times, 10 min each time, in water), mounted with vecta shield (Vector Laboratories, Inc.), observed with a fluorescence microscope (Nikon Eclipse E600 FN, with Nikon planfluor $\times 100$ lens, excitation 330 to $380 \mathrm{~nm}$ ), and imaged with a digital camera (Sony DKC ST5).

Scanning electron microscopy. Scanning electron microscopy (SEM) studies were conducted on mycelia cultured on PDA, and mature and immature leaves or calli, 3, 5, 10, and 15 days after inoculation. Leaves were placed on PDA and inoculated with a mycelial plug on either the $\mathrm{AB}$ or $\mathrm{AD}$ surface with the mycelial surface in contact with the tissue. We inspected the opposite surface for emerging hyphae. After acetone dehydration (10, 30, 50 , and $75 \%, 30$ min each, and overnight in $100 \%$ acetone), the samples were critical point dried in $\mathrm{CO}_{2}$ (EMS 850 dryer), mounted on SEM supports, sputter-coated with gold (250 to $300 \AA$, Baltzers CSD 040), examined, and photographed with a JEOL (JSM 6300F) scanning microscope at $15 \mathrm{kV}$.

Cuticle extraction. Cuticular material was extracted from four green leaves of $T$. cacao (surface area $\approx 425 \mathrm{~cm}^{2}$ ) by dipping the leaves twice in chloroform for $60 \mathrm{~s}$. The chloroform was evaporated on a rotavapor and the extract was resuspended in $1 \mathrm{ml}$ of chloroform. Six circular incisions were cut in the PDA agar, equidistant from the center and each other, using a cork borer $1 \mathrm{~cm}$ in diameter. The cuticle extract of $0,5,10,20,25$, and $30 \mu \mathrm{l}$ was diluted with chloroform to a final volume of $30 \mu$ l. Each sample was applied onto one individual circle and left overnight to allow evaporation of the chloroform. One mycelial plug was placed on the center of the plate and grown for 10 days. The samples were examined by SEM after the mycelium started to grow over the cuticular extracts.

Statistics. We analyzed the data for the effect of time, host tissue, and leaf surface on fungal growth, using analysis of variance for repeated measures (1999-2001; SAS Institute, Cary, NC) with Tukey contrasts.

\section{RESULTS}

Growth studies. SEM revealed common features in immature and mature leaves, such as the presence of multicellular and glandular trichomes and stomata. However, the AB surface contained approximately twice as many trichomes as did the AD surfaces. Stomata, which were considered to be the primary entrance for the germ tubes of $C$. perniciosa (9), were present only on the AB surface. In immature leaves, stomata present on the veins of the $A B$ surface were mature but those on the leaf lamina were in a primordial state. Despite these structural differences between the leaf surfaces, the primary mycelium did not show any significant difference in growth $(P=0.862)$ (Fig. 1) on $\mathrm{AD}$ versus $\mathrm{AB}$ surfaces.

The growth of the primary mycelium was significantly affected by the presence of host tissue ( $\mathrm{df}=3, F=139.14, P<0.0001$ ), the time period ( $\mathrm{df}=4, F=194.57, P<0.0001$ ), and the inter- 
action of time and media ( $\mathrm{df}=12, F=45.25, P=0.0001)$ (Fig. 1). In 5 days, the fungus exhibited a significantly higher growth rate on PDA alone $\left(0.25 \pm 0.04 \mathrm{~cm}^{2}\right.$ day $\left.^{-1}\right)$ than on WA $(0.16 \pm$ $0.01 \mathrm{~cm}^{2}$ day $\left.^{-1} ; P=0.0360\right)$ and the host's AB $\left(0.15 \pm 0.02 \mathrm{~cm}^{2}\right.$ day $\left.^{-1} ; P=0.0179\right)$ and AD $\left(0.15 \pm 0.02 \mathrm{~cm}^{2}\right.$ day $\left.^{-1} ; P=0.0161\right)$ leaf surfaces. On WA, the fungus ceased to grow after 10 days and had the lowest cumulative growth rate $\left(0.19 \pm 0.03 \mathrm{~cm}^{2}\right.$ day $^{-1}$; $P<0.0001)$ of all media. In the presence of the immature leaves, the lag phase was prolonged to 10 days, after which a threefold increase in the fungal growth rate was observed (Fig. 1). On PDA, the relative growth rate of the mycelium increased to $2.68 \pm$ $0.09 \mathrm{~cm}^{2}$ day $^{-1}$ by day 15 , but declined to $2.51 \pm 0.13 \mathrm{~cm}^{2}$ day $^{-1}$ by day 20 (Fig. 1). In contrast, the growth rate of the mycelium on leaf tissue continued to increase and was maximal on day 20 $\left(1.64 \pm 0.19 \mathrm{~cm}^{2}\right.$ day $^{-1}$ on $\mathrm{AB}$ and $1.5 \pm 0.2 \mathrm{~cm}^{2} \mathrm{day}^{-1}$ on $\mathrm{AD}$ surfaces).

The position of the mycelial plug on the AD surface of young leaves significantly affected mycelial growth by 10 days $(\mathrm{df}=2$, $F=24.43, P<0.0001$ ) (Fig. 2). When inoculated on the base of the leaf, the mycelium covered an area of $2.99 \pm 0.21 \mathrm{~cm}^{2}$, significantly greater than that of the midregion $\left(2.16 \pm 0.22 \mathrm{~cm}^{2}, P=\right.$ $0.0099)$ and the tip $\left(1.19 \pm 0.09 \mathrm{~cm}^{2}, P<0.0001\right)$. The growth at the tip region of the leaf also was significantly less than that of the midregion $(P<0.0026)$. Growth of the mycelium in the midregion of the AD leaf surface on WA $\left(2.16 \pm 0.22 \mathrm{~cm}^{2}\right)$ (Fig. 2) was comparable to that on PDA $\left(1.94 \pm 0.17 \mathrm{~cm}^{2}\right)$ (Fig. 1), indicating that the effect on growth was a response to the host tissue, not to the PDA medium.

The immature leaves began to degenerate earlier when inoculated on the $\mathrm{AB}$ surface than after infection on the $\mathrm{AD}$ surface. Regardless of the inoculated surface, the leaves started to wrinkle after 5 days and became necrotic after 12 to 15 days, which coincided with increased fungal growth. The continued degradation of leaves was accompanied by exudates of golden yellowish color in the medium. Growth of the primary mycelium on mature leaves was affected by profuse endophyte contamination and, thus, not quantified. Although mature leaves developed necrotic lesions after fungal inoculation, the leaves did not completely disintegrate within the 20-day study period. The primary mycelium generated from spores and the ATCC cultures resulted in similar mat-like growth of creamy and glossy appearance on PDA as well as on cacao leaves, whereas the mycelium on WA appeared hyaline and dull.

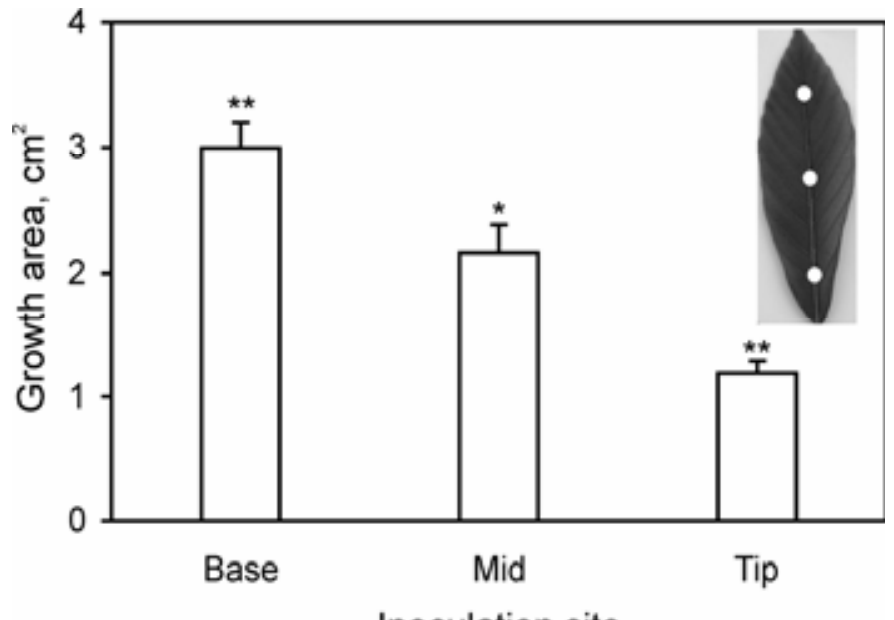

Inoculation site

Fig. 2. Growth rate of mycelial plugs of Crinipellis perniciosa that were inoculated onto the adaxial surfaces of young leaves of Theobroma cacao decreased from the base of leaves toward the tip. The positional effect was significant $(\mathrm{df}=2, F=24.43, P<0.0001)$ within 10 days. The insert shows the sites of inoculation and the bars represent standard errors of means. Asterisks indicate $P<0.0001$ (**) or $P<0.05$ (*) between the positions of the leaf.
Phase transition in relation to growth. Epifluorescence microscopy of DAPI- and calcofluor-stained mycelia on WA (5 to 10 days old) showed plasmolyzed and, rarely, binucleate hyphal cells. On PDA monokaryotic, dikaryotic, and occasional enucleate conditions were observed within the same colony, which is indicative of incomplete phase transition. Furthermore, only the peripheral region with actively growing hyphal tips was monokaryotic (Fig. 3A). Older mycelium close to the site of inoculation and regions at least $3 \mathrm{~cm}$ away from the growing tips contained two nuclei (Fig. 3B). Dikaryotization was initiated 5 days after inoculation on PDA, the beginning of the exponential growth phase. The retention of the uninucleate condition in the peripheral cells of the mycelium on PDA allowed further subculture of primary mycelium.

Mycelia growing on either surface of immature leaves underwent phase transition after 10 to 12 days, which produced thin, hyaline secondary hyphae with clamp connections. In mature leaves, clamp formation occurred within 3 to 5 days. However, clamp formations were absent from the growing tips in any cul-



Fig. 3. Epifluorescence images of potato dextrose agar (PDA)-grown mycelia of Crinipellis perniciosa after staining with 4',6-diamidino-2-phenylindole for nuclei and calcofluor for the cell walls. A, Until 5 days on PDA or 10 to 12 days on immature cacao leaves, the mycelium was in a unikaryotic state. $\mathbf{B}$, Secondary mycelia with dikaryotic clamp formation were seen on older cultures, 10 days or more on PDA, more than 12 days on immature tissues, and 3 to 5 days on mature tissue; $\mathrm{n}=$ nucleus, $\mathrm{s}=$ septum, and $\mathrm{cf}=$ clamp formation. $\mathrm{Bar}=10 \mu \mathrm{m}$. 


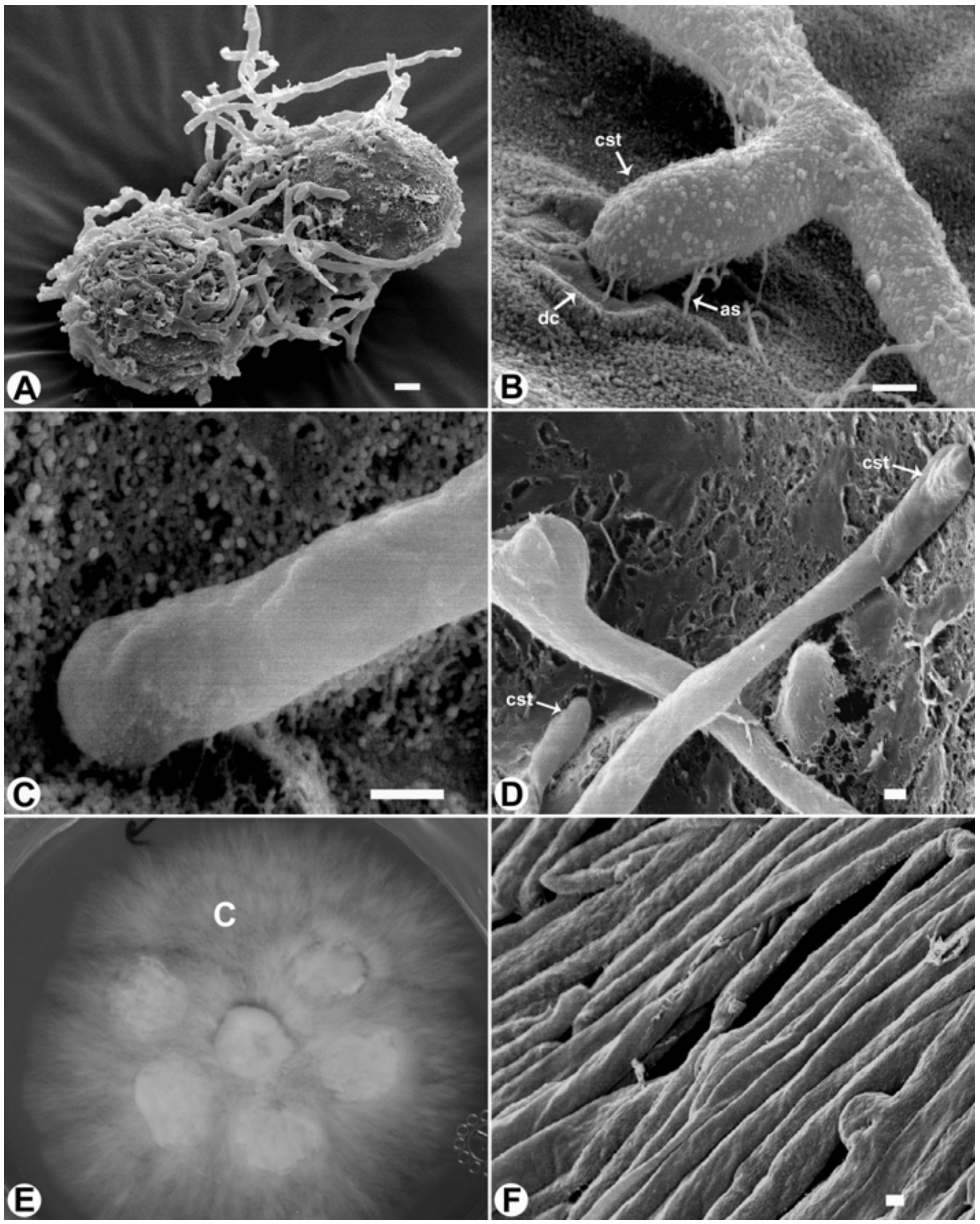

Fig. 4. Morphological changes in the hyphae of Crinipellis perniciosa on interaction with the tissues of Theobroma cacao. A, Leaf callus of T. cacao was encircled by primary mycelium of $C$. perniciosa but no penetration was visible. Bar $=10 \mu \mathrm{m}$. B, Club-shaped tip formation at the site of penetration of $T$. cacao leaf by a single hypha of $C$. perniciosa; $\mathrm{dc}=$ localized cuticle disintegration, as = adhesive structures, and cst = club-shaped tip. $\mathbf{C}$, Hypha show rounded tips on agar or when not engaged in penetration. D to F, Primary mycelium on exposure to cuticle extract. D, Induction of club-shaped tips. E, Dense mat-like growth of mycelium was observed on agar that was enriched with cuticular material. Growth over control agar (C, without cuticular extract) was less dense. F, Rhizomorphlike structures with parallel hyphae and clamps. B, C, D, and $\mathbf{F}, \mathrm{Bar}=1 \mu \mathrm{m}$. 
ture conditions. Binucleate cells were longer than uninucleate primary mycelial cells and their nuclei appeared spindle shaped (Fig. 3B).

Pathogenicity of the primary mycelium. Callus cells were encircled by the biotrophic mycelium but penetration was not observed (Fig. 4A). Upon infection, callus cells turned dark brown in 5 days and produced brownish exudates. Most of the calli died within 2 weeks and clamp formation was noticed only 15 days after infection. On host tissue, the primary mycelium formed club-shaped tips prior to penetration (Fig. 5) and caused localized disintegration of the surface at the site of penetration (Fig. 4B). We also observed thin, thread-like connections between the hyphal surface and the substrate. Although club-shaped tips caused some deformation in the cuticle (Fig. 4B), haustoria or appressoria were not observed. When cultured on agar medium, the tips of the primary mycelium remained round (Figs. 4C and 5 ); however, the application of cuticle extract induced clubshaped tips (Fig. 4D). The cuticle extract did not affect the fungal linear growth rate but caused denser, mat-like growth (Fig. 4E). The hyphae tended to elongate unbranched and parallel to each other, similar to rhizomorphs (Fig. 4F).

The primary mycelium penetrated both immature and mature leaves independent of stomata and irrespective of the surface of inoculation. In general, the hyphae did not show any tropic response or preferential growth toward open stomata (Fig. 6A). Of 35 sites of pathogen entry into the host tissue (Fig. 5), stomatal entry was observed only once. Mycelial colonization was common along the veins (Fig. 6B) and induced blister formation on the leaf surface (Fig. 6B) in regions of profuse mycelial growth. Penetration with numerous club-shaped tips was common at the base of foliar (Fig. 6C) and glandular trichomes (Fig. 6D). The hyphae typically reached the opposite surface in 3 to 5 days, irrespective of the surface of inoculation (Fig. 6E), and eventually colonized the entire leaf tissue. Penetration sites commonly were used by multiple hyphae for entry and exit (Fig. 6B to F). Five days postinoculation, the mycelium exhibited inter- and intracellular growth (Fig. 6F) and induced localized necrosis. The mycelium showed clamp connections 12 to 15 days postinoculation (Fig. 6G). At this stage of infection, complete digestion of the inner mesophyll cells was noticed, leaving only fibrillar debris and cuticle. The cuticle was ruptured but not digested. In mature leaves, tissue degeneration was not noticeable until 10 to 12 days despite the emergence of dikaryotic hyphae within 3 to 5 days of infection.

\section{DISCUSSION}

C. perniciosa is a hemibiotrophic basidiomycete, yet lacks a clear temporal and spatial delineation between its primary and secondary mycelial stage. Furthermore, the specific effects of each fungal phase on the host are unknown. The hypertrophy during primary infection and tissue death during the secondary phase make the witches' broom disease an interesting system to study host-pathogen interaction. In the natural habitat of $T$. cacao, disease symptoms appear in the meristematic parts of the host, such as floral cushions and new flushings (4), although other parts are equally likely to be exposed to basidiospores. The interactions of the fungus with cell and tissue cultures were studied previously $(8,23)$, but no reference was made as to the influence of mature tissues on fungal growth. In the research reported here, we addressed how the pathogen responds to meristematic and nonmeristematic tissues and whether only immature regions are susceptible to pathogen entry and infection by investigating the growth pattern of the developing primary mycelium on host leaf tissue.

Developmental changes in the primary mycelium. The primary mycelium of $C$. perniciosa entered and infected mature and immature leaves, irrespective of the inoculated surface. The ob- served differential phase transition likely was influenced by a combination of nutrients, stress, metabolites, and host defense mechanisms.

Nutrient dependency for successive crops of fruiting bodies during the saprophytic stage of $C$. perniciosa has been well documented (12). We observed that the phase transition was not achieved on WA-grown cultures where the growth of the primary mycelium was minimal (Fig. 1). Additionally, on host tissue, 10 days were required by the fungus to grow to a size equivalent to that of 5-day culture on PDA. After a common growth area or biomass was achieved (Fig. 1) by the primary mycelium, phase transition occurred (Fig. 3), suggesting nutrient dependency during the biotrophic stage. The nutrient dependency is further supported by the different growth rates on young leaves (Fig. 2). Additionally, the presence of endophytes (3) is likely to affect the growth of $C$. perniciosa and may accelerate phase transition, similar to plants that accelerate their transition to the generative phase in response to abiotic $(20)$ and biotic stresses $(1,18)$.

In addition to the nutrient requirements, the incomplete phase transition in PDA cultures also suggests dependency on host cues, such as hormones or metabolites, as suggested by Evans (8). C. perniciosa-infection of young leaves stimulates synthesis of caffeine that inhibits fungal growth (2). Thus, caffeine, that often induces binucleate cells (15), or related alkaloids may have attributed to phase transition. Hammerstone et al. (11) reported a declining gradient of caffeine from the youngest to oldest leaf within the same branch of IH-220 cacao. The difference in growth achieved by the fungus based on its position on leaf (Fig. 2) may have resulted from a similar gradient of a metabolite or hormone within a leaf. Additionally, the observed wrinkling of leaves, exudation, and necrosis may represent a defense response and restrict fungal growth or phase transition.

The difficulty of generating basidiocarps in vitro in the absence of the host material also indicates host-dependent signals for C. perniciosa to complete its life cycle. Thus, the fungus may retain uninucleate hyphae or primary mycelium and not complete the phase transition in the absence of such host-specific signals. This incomplete transition might explain recurring infections on the same tree in the natural habitat.

Pathogenicity of primary mycelium. Frias et al. (9) observed strong tropic response by the germ tubes toward stomata. However, established mycelium did not exhibit tropic responses. Because of their premature state, stomata may not be the main source of entry for the primary mycelium. The trichomes attracted infection $(17,19)$ and were common sites for pathogen entry

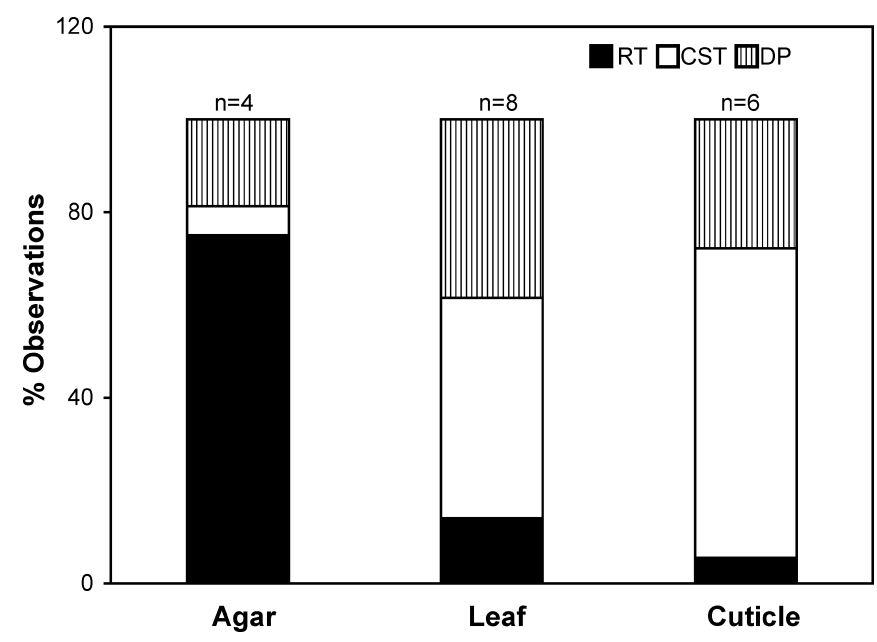

Fig. 5. Relative occurrence of club-shaped tips (CST), direct penetration (DP), or round-tipped (RT) hyphae as a consequence of exposure to host leaves or cuticle extracts. RT hyphae were observed mostly on agar and in the absence of penetration. The observations were derived from independent plates. 


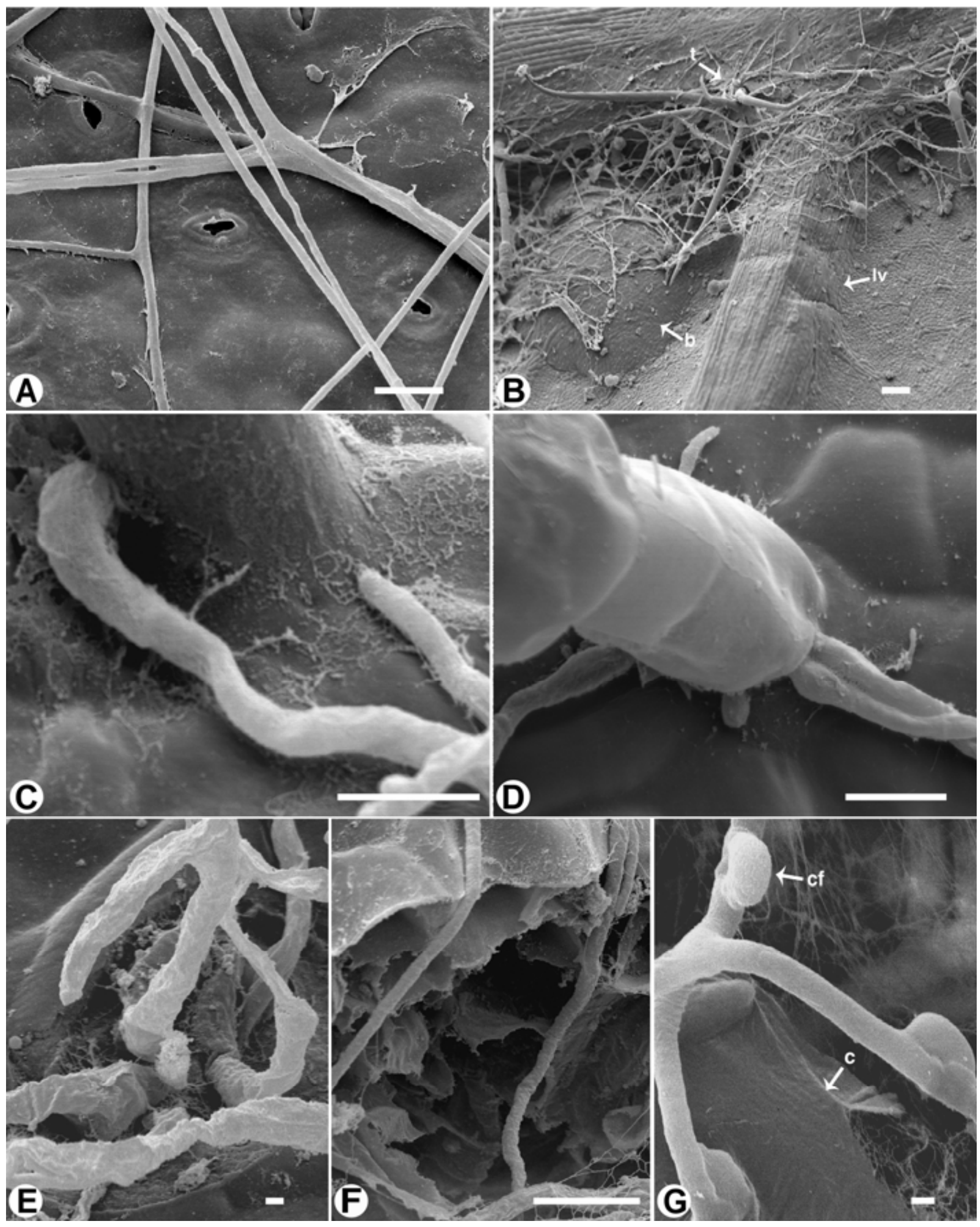

Fig. 6. Growth pattern of the primary mycelium of Crinipellis perniciosa on Theobroma cacao leaves. A, Hyphae typically ignored open stomata as possible entry points. Bar $=10 \mu \mathrm{m}$. B, Profuse growth of the mycelium along lateral veins (lv) and trichomes (t) resulted in blisters (b). Bar $=100 \mu \mathrm{m}$. C, Club-shaped tips at the base of the foliar and (D) glandular trichomes suggest impending penetration. Bar $=10 \mu \mathrm{m}$. E, Hyphae emerged from the opposite surface within 3 days and the exit site showed damage to the leaf cuticle. Bar $=1 \mu \mathrm{m}$. F, The intercellular hyphae inside a leaf typically were accompanied by numerous filaments that resembled thread-like connections between hyphae and cuticle. Bar $=10 \mu \mathrm{m}$. G, Digested young leaves were characterized by remnants of cuticle and the formation of clamps, indicative of phase transition; $\mathrm{c}=$ cuticle, $\mathrm{cf}=$ clamp formation. $\mathrm{Bar}=1 \mu \mathrm{m}$. 
(Fig. 5C and D). The profuse infection and penetrations at veins and trichomes may result from higher availability of nutrients at those sites or modified (stretched) cuticle. However, glandular trichomes contain essential oils that may be involved in defensive responses $(31,32)$. Further testing is required to determine if the abundance of glandular trichomes on the $\mathrm{AB}$ surface of $T$. cacao leaves affects the degeneration of leaves. Blister formation as a result of hyperplasia and hypertrophy of infected tissues (6) also attests to the pathogenicity of primary mycelium.

Most phytopathogenic fungi form specialized penetration organs such as appressoria which, in some organisms, are capable of producing high turgor pressure ( $>8 \mathrm{MPa}$ ) (13) and assist in mechanically penetrating the host tissue. In $C$. perniciosa, the attachment of the club-shaped tips to the host tissue via extracellular material suggests a possible role of these adhesive structures similar to that of appressoria. The absence of hyphal penetration and club-shaped tips on agar or callus suggests that these structures are initiated by signals that are absent in callus cells and nutritive medium. These signals could be derived from the cuticle because neither the callus nor chemical media have cuticle-like compounds, and penetrating tips were observed when the fungus was exposed to host cuticle extract (Figs. 4 and 5). The response of the primary mycelium to cuticle extract suggests a possible role of the cuticle in host recognition, similar to that of avocado wax-induced appressorium formation in Colletotrichum gloeosporiodes (16). Fungal spores are likely to perceive the plant surface chemically and induce penetration of the host barriers. Although detaching of the leaves may have altered natural defense responses, wounds are unlikely to result from processing or sanitation and, therefore, are unlikely to contribute to penetration as was suspected by Frias et al. (9).

Our data suggest that the primary mycelial stage is not ephemeral and that phase transition is correlated with nutrient accumulation and the developmental stage of the host. In addition, other endophytes may compete for space and resources or antagonize Crinipellis perniciosa directly. Further studies will elucidate the significance of these endophytes in correlation with phase transition and, thus, possible biocontrol for the witches' broom disease. Surface signaling is likely to play an essential role in cacao invasion by the primary mycelium of $C$. perniciosa. The complex but repeatable responses in our experiments suggest that the use of primary mycelium is a suitable experimental alternative for commonly used spore inoculation. We have initiated investigations on host-specific cues and physiological and genetic responses leading to phase transition and information exchange between T. cacao and C. perniciosa.

\section{ACKNOWLEDGMENTS}

This research was supported by Graduate Student Organization of UL Lafayette (2001-03), DOE grant DE-FG02-97ER12220 and NASA grant NAG10-0190. We thank P. Hebbar of Mars Inc., for the spores of Crinipellis perniciosa and B. Bailey (USDA-ARS, Beltsville, MD) for his input on this manuscript.

\section{LITERATURE CITED}

1. Agnew, P., Koella, J. C., and Michalakis, Y. 2000. Host life history responses to parasitism. Microbes Infect. 2:891-896.

2. Aneja, M., and Gianfagna, T. 2001. Induction and accumulation caffeine in young, actively growing leaves of cocoa (Theobroma cacao L.) by wounding or infection with Crinipellis perniciosa. Physiol. Mol. Plant Pathol. 59:13-16.

3. Arnold, A. E., Mejia, L. C., Kyllo, D., Rojas, E. I., Maynard, Z., Robbins, N., and Herre, E. A. 2003. Fungal endophytes limit pathogen damage in a tropical tree. Proc. Natl. Acad. Sci. USA 100:5649-15654.

4. Baker, R. E. D., and Holliday, P. 1957. Witches' broom disease of cacao (Marasmius perniciosus Stahel). Commonw. Mycol. Inst. Phytopathol. Pap. 2:1-42.
5. Cronshaw, D. K., and Evans, H. C. 1978. Witches' broom disease of cocoa Crinipellis perniciosa in Ecuador. II. Methods of infection. Ann. Appl. Biol. 89:193-200.

6. Dabydeen, S., and Sreenivasan, T. N. 1989. Occurrence, anatomy and morphology of "blisters" on cocoa seedlings inoculated with Crinipellis perniciosa. Plant Dis. 73:481-484.

7. Delgado, J. C., and Cook, A. A. 1976. Nuclear condition of the basidia, basidiospores and mycelium of Marasimus perniciosus. Can. J. Bot. 54:66-72.

8. Evans, H. C. 1980. Pleomorphism in Crinipellis perniciosa, causal agent of witches' broom disease of cocoa. Trans. Br. Mycol. Soc. 74: 515-523.

9. Frias, G. A., Purdy, L. H., and Schmidt, R. A. 1991. Infection biology of Crinipellis perniciosa on vegetative flushes of cacao. Plant Dis. 75:552556.

10. Frias, G. A., Purdy, L. H., and Schmidt, R. A. 1995. An inoculation method for evaluating resistance of cacao to Crinipellis perniciosa. Plant Dis. 79:787-791.

11. Hamerstone, J. E., Romanczyk, L. J., and Aitken, W. M. 1994. Purine alkaloid distribution within Herrania and Theobroma. Phytochemistry 35:1237-1240.

12. Hedger, J. 1985. Tropical agarics, resource relations and fruiting periodicity. Pages 41-86 in: Developmental Biology of Higher Plants. D. Moore, L. A. Casselton, D. A. Wood, and J. C. Frankland, eds. Cambridge University Press, Cambridge.

13. Howard, R. J., Ferrari, M. A., Roach, D. H., and Money, N. P. 1991. Penetration of hard substrates by a fungus employing enormous turgor pressures. Proc. Natl. Acad. Sci. USA 88:11281-11284.

14. ICCO 2003. Press releases. Q. Bull. Cocoa Stat. 29:315.

15. Kihlman, B. A., and Anderson, H. C. 1987. Effects of caffeine on chromosomes in cells of higher organisms. Rev. Environ. Health 7:279-382.

16. Kolattukudy, P. E., Rogers, L. M., Li, D. X., Hwang, C. S., and Flaishman, M. A. 1995. Surface signaling in pathogenesis. Proc. Natl. Acad. Sci. USA 92:4080-4087.

17. Kontaxis, D. G. 1962. Leaf trichomes as avenues for infection sites for Corynebacterium michiganense. Phytopathology 52:1306-1307.

18. Korves, M. T., and Bergelson, J. 2003. A developmental response to pathogen infection in Arabidopsis. Plant Physiol. 133:339-347.

19. Layne, R. E. C. 1967. Foliar trichomes and their importance as infection sites for Corynebacterium michiganense on tomato. Phytopathology 57:981-985.

20. Levy, Y. Y., and Dean, C. 1998. The transition of flowering. Plant Cell 10:1973-1989.

21. Mendgen, K., and Deising, H. 1993. Infection structures of fungal plant pathogens: A cytological and physiological evaluation. New Phytol. 124:193-213.

22. Murashige, T., and Skoog, G. 1962. A revised medium for rapid growth and bioassays with tobacco tissue cultures. Physiol. Plant. 15:473-497.

23. Muse, R. B., Collin, H. A., Isaac, S., and Hardwick, K. 1996. Effects of the fungus Crinipellis perniciosa, causal agent of witches' broom disease, on cell and tissue cultures of cocoa (Theobroma cacao L.). Plant Pathol. 45:145-154.

24. Orchard, J., Collin, H. A., Hardwick, K., and Isaac, S. 1994. Changes in morphology and measurement of cytokinin levels during the development of witches' brooms on cocoa. Plant Pathol. 43:65-72.

25. Pegus, J. E. 1972. Aspects of the host-parasite relationship in the Theobroma cacao L./Marasimus perniciosus Stahel disease complex. M.Sc. thesis. University of West Indies, Trinidad.

26. Purdy, L. H., and Schmidt, R. A. 1996. Status of cacao witches' broom: Biology, epidemiology, and management. Annu. Rev. Phytopathol. 34:573-594.

27. Rocha, H. M., and Wheeler, B. E. J. 1985. Factors influencing the production of basidiocarps and the deposition and germination of basidiospores of Crinipellis perniciosa, the causal fungus of witches' broom on cocoa (Theobroma cacao). Plant Pathol. 34:319-328.

28. Singer, R. 1942. A monographic study of the genera Crinipellis and Chaeltocalathus. Lilloa 8:441-513.

29. Sreenivasan, T. N., and Dabydeen, S. 1989. Modes of penetration of young cocoa leaves by Crinipellis perniciosa. Plant Dis. 73:478-481.

30. Stahel, G. 1915. Marasmius perniciosus nov. spec., the cause of the krulloten disease of cacao in Suriname. Dep. van den Landbouw, Suriname, Bull. No. 33:25.

31. Traw, M. B., and Bergelson, J. 2003. Interactive effects of jasmonic acid, salicylic acid, and gibberellin on induction of trichomes in Arabidopsis. Plant Physiol. 133:1367-1375.

32. Wagner, G. J., Wang, E., and Shepherd, R. W. 2004. New approaches for studying and exploiting an old protuberance, the plant trichome. Ann. Bot. 93:3-11. 